\title{
PROPOSTA DE INTEGRAÇÃO ENTRE BIM E ACV UTILIZANDO COMPOSIÇÕES DE SERVIÇO
}

\author{
BIM AND LCA INTEGRATION PROPOSAL USING SERVICE COMPOSITIONS
}

\author{
Druno Thiago Rego Valeriano \\ Silva ${ }^{1}$ \\ (D) Maryangela Geimba de Lima ${ }^{2}$ \\ ${ }^{1}$ Instituto Tecnológico de Aeronáutica, São \\ José dos Campos, SP, Brasil, \\ brunothiagorvs@gmail.com \\ 2 Instituto Tecnológico de Aeronáutica, \\ São José dos Campos, SP, Brasil, \\ magdlima@ita.br
}

Contribuição dos autores:

BTRVS: conceituação, curadoria de dados, análise formal, investigação, metodologia, programas, validação, visualização, escrita - rascunho original, escrita - revisão e edição. MGL: conceituação, análise formal, investigação, metodologia, administração de projetos, supervisão, validação, visualização, escrita - rascunho original, escrita - revisão e edição.

Fomento: Conselho Nacional de Desenvolvimento Científico e Tecnológico Declaração de conflito: nada foi declarado.

Editor Responsável:

Vanessa Gomes da Silva (iD

\begin{abstract}
Resumo
A indústria da construção civil vem apresentando esforços buscando alcançar os avanços tecnológicos necessários para garantir o constante aumento na qualidade das obras e, consequentemente, melhoria na sustentabilidade de seus produtos. Nesse sentido, a tecnologia Building Information Modeling se destaca como uma forma de permitir que diversas equipes e sistemas se comuniquem e agreguem informações a um projeto. Por outro lado, a Avaliação de Ciclo de Vida apresenta um método sistemático para a identificação do desempenho ambiental de produtos, com a dificuldade, porém, de ser necessária a compilação e avaliação de uma quantidade relativamente grande de dados, tornando o trabalho do avaliador extremamente complexo e demorado. Portanto, a recente busca pela integração desses dois assuntos aparece como uma alternativa viável para garantir que engenheiros, arquitetos e construtores tenham acesso a informações importantes durante qualquer fase no desenvolvimento do projeto e possam, assim, tomar decisões melhores, baseadas em dados providos por sistemas computacionais. Visando essa integração, o presente trabalho apresenta uma abordagem que permite a utilização de bancos de composições de custos para o levantamento de materiais e de inventários de ciclo de vida em ambientes BIM, facilitando a utilização de indicadores ambientais em conjunto com custos. Foram utilizadas rotinas de programação para permitir a conversão e organização dos dados, além da personalização de um software multifunção BIM para permitir a quantificação dos elementos. A abordagem é então demonstrada em um estudo de caso de seleção de materiais, de forma exemplar, demonstrando o funcionamento do que foi desenvolvido.
\end{abstract}

Palavras-chave: Building Information Modeling. Avaliação de Ciclo de Vida. Aquecimento global. Custos.

\begin{abstract}
The civil construction industry has been making efforts to achieve the technological advances necessary to guarantee the constant increase in the quality of buildings and, consequently, improve the sustainability of its products. In this sense, Building Information Modeling technology stands out to allow different teams and systems to communicate and add information to a project. On the other hand, the Life Cycle Assessment presents a systematic method for identifying the environmental performance of products, with the difficulty, however, of the need to compile and evaluate a relatively large amount of data, making the evaluator's work extremely complex and time-consuming. Therefore, the recent research for the integration of these two issues appears as a viable alternative to ensure that engineers, architects, and builders have access to important information during any phase in the development of the project and can, thus, make better decisions based on data provided by computational systems. Aiming at this integration, the present work presents an approach allowing the use of cost composition databases to estimate the bill of materials and life cycle inventories simultaneously in BIM environments, facilitating environmental indicators and costs parameters in parallel. Programming scripts were used to allow the conversion and organization of the data and the customization of multifunction BIM software to quantify building elements. The approach is then demonstrated in a case study of material selection, as an example, showing the functioning of what was developed.
\end{abstract}

Keywords: Building Information Modeling. Life Cycle Assessment. Global warming. Costs.

How to cite this article:

SILVA, B. T. R. V.; LIMA, M. G. de. Proposta de integração entre BIM e ACV utilizando composições de serviço. PARC

Pesquisa em Arquitetura e Construção, Campinas, SP, v. 12, p. e021017, 2021. DOI:

http://dx.doi.org/10.20396/parc.v12i00.8659910 


\section{Introdução}

A indústria da construção vem passando por avanços tecnológicos constantes, sempre objetivando aumentos na produtividade, qualidade dos projetos e, consequentemente, construção de edificações cada vez mais eficientes. Desde a evolução para a utilização de ferramentas computadorizadas para o auxílio na elaboração de desenhos, $C A D$, até a adoção da mais recente da tecnologia conhecida como Building Information Modeling (BIM), projetistas buscam, na automação baseada em computadores, formas de reduzir a carga de trabalho em tarefas repetitivas assim como facilitar a identificação de erros ou a execução de levantamentos de quantitativos.

BIM pode ser definido como uma representação digital de toda a construção, ou seja, é um modelo digital contendo todas as características físicas e funcionais de uma obra, representando-a tanto graficamente quanto não graficamente, armazenando informações relevantes para simulações, extração de quantidade e tomadas de decisões. Além disso, a utilização do BIM objetiva a comunicação e troca de informações entre equipes e sistemas, buscando a entrega integrada do projeto e desfragmentando a indústria da construção, tradicionalmente conhecida por erros, retrabalhos e falta de diálogo constantes entre os envolvidos em um projeto (AZHAR, 2011; BARLISH; SULLIVAN, 2012; NATIONAL INSTITUTE OF BUILDING SCIENCES, 2019; THE AMERICAN INSTITUTE OF ARCHTECTS, 2007).

Nesse sentido, a utilização da tecnologia BIM vem sendo fruto de estudos recentes, buscando desmitificar sua utilização e demonstrar quais são as vantagens do seu uso, desde a identificação prévia de interferência entre elementos do modelo (RADKE; WALLMARK; TSENG, 2009), levantamento de quantitativos e orçamentação (KEHILY, 2017), planejamento da construção e de serviços (HU; ZHANG; DENG, 2008), gerenciamento de instalações (NEATH; HULSE; CODD, 2014) e, mais recentemente, como uma forma de auxiliar na avaliação de desempenho ambiental das edificações (WONG; ZHOU, 2015).

Além de prover informações que possibilitem a avaliação energética das construções, a partir da entrada das informações sobre materiais, espessura de sistemas de isolamento, localização geográfica e, inclusive, previsão de ocupação e usos (KENSEK; KUMAR, 2008), é possível aplicar BIM na quantificação de materiais, permitindo estimar entradas, saídas e impactos potenciais de todo o sistema construtivo, tarefa necessária durante a realização da Avaliação do Ciclo de Vida (ACV) da obra (BARROS; SILVA, 2016).

A ACV é uma metodologia utilizada para se avaliar de forma quantitativa os impactos ambientais associados ao uso de materiais, energia e transporte durante $o$ desenvolvimento de produtos. É utilizada por diversas indústrias e se apresenta como uma abordagem sistemática para a quantificação de impactos, se baseando em quatro etapas, conforme normatizado internacionalmente por padrões ISO (INTERNATIONAL ORGANIZATION FOR STANDARDIZATION, 2006a, 2006b), que envolvem a definição do objetivo e escopo, a análise de inventário e a avaliação do impacto de ciclo de vida. Todas as etapas devem ser interpretadas e a busca por melhoria, contínua.

O principal obstáculo da utilização do ACV em produtos da indústria da construção se encontra na inevitável complexidade desses. Construções são, por natureza, produtos multidisciplinares, envolvendo diversas áreas do conhecimento, desde a arquitetura até a avaliação estrutural, o dimensionamento de sistemas de tubulações hidráulicas e elétricas, dentre outros que, ao final, precisam ser quantificados, avaliados e compatibilizados (SOUST-VERDAGUER; LLATAS; GARCÍA-MARTÍNEZ, 2016). Portando, a integração entre ACV e BIM aparece como uma solução que se propõe a reduzir o trabalho de quantificação, reduzir erros e garantir acesso a uma vasta quantidade de 
dados de diferentes disciplinas de forma que a avaliação de ciclo de vida possa ser realizada e interpretada, guiando os responsáveis na seleção de materiais e técnicas, inclusive durante fases iniciais de desenvolvimento, quando as decisões tomadas tem maior poder de dirigir no sentido desejado (SOUST-VERDAGUER; LLATAS; GARCÍAMARTÍNEZ, 2017). Com isso, o presente trabalho pretende apresentar uma forma de realizar tal integração. Foi utilizado software BIM multifunção (SACKS et al., 2018), capaz de integrar diversas disciplinas e realizar a quantificação de materiais de forma dinâmica. Para tal, desenvolveu-se bancos de dados e rotinas de programação visando a automação na conversão e inserção das informações necessária entre as diferentes plataformas utilizadas.

Com isso, esse estudo objetiva apresentar aos pesquisadores de Avaliação de Ciclo de Vida com foco em produtos da construção ou profissionais da indústria da construção uma forma de utilizar softwares que empregam a tecnologia Building Information Modeling como ferramenta de auxílio no levantamento de quantitativos, atribuição de desempenho ambiental e tomada de decisão baseada em indicadores diversos, incluindo custos. Apesar de, obviamente, a qualidade do levantamento de quantitativos depender da maturidade do projeto em avaliação, a metodologia apresentada é passível de utilização desde as fases iniciais de projeto.

\section{Fundamentação}

A adoção da Avaliação de Ciclo de Vida no setor da construção vem apresentando crescimento constante ao longo dos anos, conforme indica a Figura 1. Os dados para cada ano foram obtidos utilizando a query string "((Life Cycle Assessment OR LCA) AND ((build* OR construct* OR architecture) OR ((building) AND (materials OR HVAC OR Systems))))" na base Engineering Village, filtrando para artigos de periódicos em inglês, conforme sugerido por Geng et al. (2017). Esse crescimento, por sua vez, explicita a tendência para a aceitação da ACV como uma ferramenta auxiliar no desenvolvimento de projetos sustentáveis, permitindo a análise dos possíveis impactos ambientais associados às decisões tomadas pelos profissionais envolvidos. Não diferente, a proposta de desenvolver sistemas ou métodos capazes de facilitar a integração entre softwares desenvolvidos para atender à tecnologia BIM e a metodologia ACV também vem apresentando crescimento constante, conforme indicado por Barros e Silva (2016).

Figura 1 - Crescente número de publicações voltadas para o estudo da utilização da avaliação de ciclo de vida para edificações (Levantam ento conforme sugerido por Geng et al. (2017): os termos de busca incluíram "Life Cycle assessment" ou "LCA" e "building" ou "construction", além de considerar aspectos de construções, como sistemas de ar-condicionado e tubulações. Apenas artigos em periódicos foram contabilizados. Dados levantados em abril de 2021, portanto, representam o último ano não finalizado)

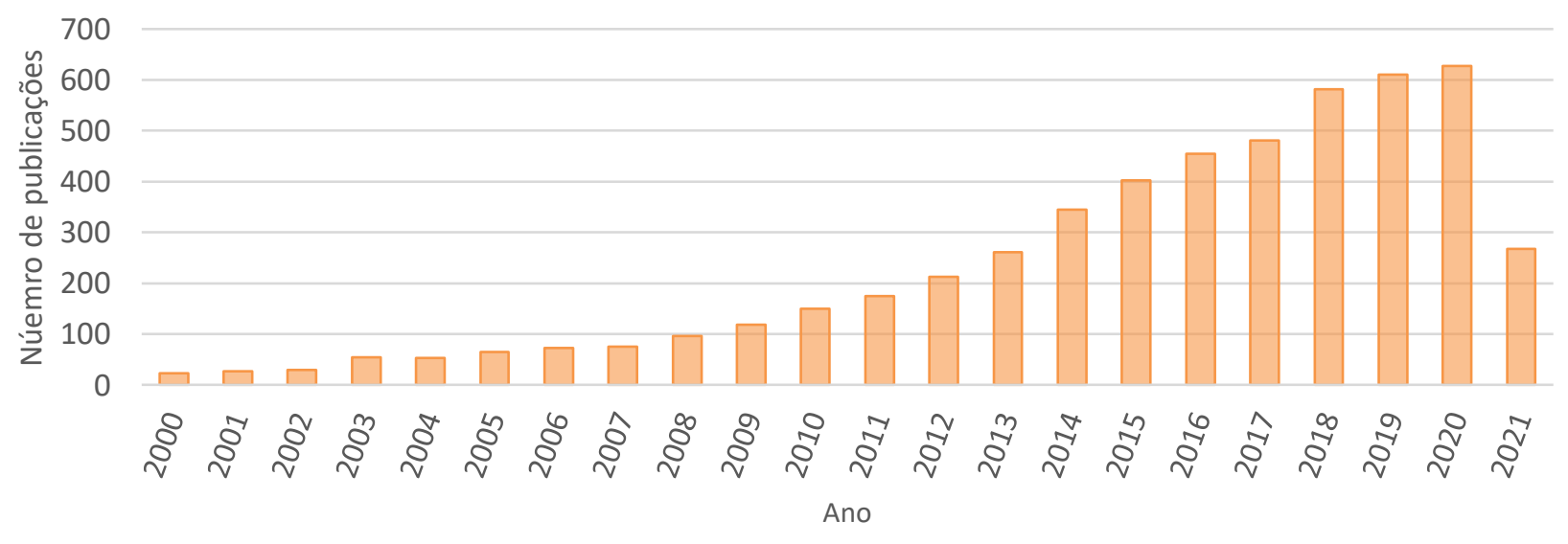

Fonte: os autores. 
Dentre essa crescente disponibilidade de artigos e estudos relacionando construções e métodos de avaliação de impactos ambientais, é comum identificarmos a adoção de abordagens distintas. A importância em se categorizar as diferentes abordagens identificadas se justifica em definir parâmetros comuns de classificação de materiais, métodos construtivos ou, até mesmo, edificações como um todo. A primeira categoria, avaliação da construção como um todo, compreende estudos cujo objetivo envolve avaliar o processo construtivo de forma holística, ou seja, identificando o impacto associado às diferentes fases de uma obra (construção, uso e desmonte), como se distribui o consumo energético e como técnicas e soluções de projeto influenciam no desempenho ambiental da instalação objeto de estudo. Outra abordagem envolve a avaliação dos materiais selecionados. Cada material utilizado numa construção tem uma história. A partir da extração até a disposição final (cradle to gravel), diversos processos estão envolvidos para que um material possa ser utilizado em obras, desde o transporte entre diferentes indústrias, responsáveis pelo processamento e transformações dos materiais, até cozimentos e curas, incluindo também a energia despendida na montagem, utilização e manutenção até, finalmente, o fim da vida útil desse material, que pode ser reciclado ou descartado. Tudo isso deve ser considerado na identificação dos impactos associados com a seleção de um determinado material (ORTIZ; CASTELLS; SONNEMANN, 2009).

Portanto, o levantamento de todas as informações necessárias pode se tornar uma tarefa rapidamente trabalhosa para o profissional responsável pela ACV de uma edificação completa. Com isso considerado, apresentam-se na literatura, estratégias que propõem utilizar modelos BIM como fonte de algumas das informações necessárias para que avaliações de edificações possam ser mais facilmente realizadas.

O estudo apresentado por llhan e Yaman (2016) demonstrou a utilização de uma ferramenta denominada Green Building Assessment Tool (GBAT). A ferramenta permite que um modelo extraído do software de modelagem BIM ArchiCAD seja avaliado quanto a classificação para o sistema de avaliação ambiental Building Research Establishment Environmental Assessment Method (BREEAM). Porém, como discutido no estudo, ainda é necessário que usuários façam alterações manuais no modelo, além de ser necessária a modificação de alguns parâmetros, o que pode ser visto como dificultador da utilização da proposta por alguns profissionais que já possuam um fluxo de trabalho bem definido (ILHAN; YAMAN, 2016).

Ao contrário do proposto por ferramentas independentes, como o GBAT, é comum que pesquisadores utilizem interfaces de programação de aplicativos (API) disponibilizadas por desenvolvedoras de softwares de modelagem BIM como alternativa para o desenvolvimento de ferramentas de integração com ACV. Essa estratégia tem como vantagem a celeridade com que essas ferramentas, normalmente conhecidas como addins, são desenvolvidas, uma vez que toda a interface de usuário e algumas funções podem ser aproveitadas do software utilizado como base (OTI et al., 2016).

Uma solução de integração BIM cuja utilização de add-ins é a apresentada por Inyim, Rivera e Zhu (2015), que demonstraram o desenvolvimento do SimulEICon. Com base na API do software BIM Revit, SimulEICon permite a otimização do projeto tendo como critérios o tempo estimado para a construção, o custo e a emissão de $\mathrm{CO}_{2}$ prevista. A ferramenta permite ainda que limites sejam estipulados entre os critérios, tornado possível que o projetista defina um orçamento ou uma medida máxima para a emissão de carbono prevista, otimizando o projeto com base na seleção de alternativas entre as possíveis combinações de materiais cadastrados no modelo (INYIM; RIVERA; ZHU, 2015). 
É necessário, porém, realizar maiores validações a fim de garantir que a integração entre BIM e ACV garanta resultados tão precisos quanto aqueles realizados por ferramentas dedicadas, como GaBi (SPHERA, 2020), SimaPro (SIMAPRO, 2020) ou OpenLCA (GREENDELTA, 2020). É possível que add-ins não possibilitem os níveis necessários de configuração de parâmetros para que o usuário possa realizar considerações necessárias com o objetivo de se obter resultados válidos. Outro ponto importante de se considerar é a relação intrínseca entre o que é modelado e o que é quantificado em um projeto BIM. Por exemplo, fôrmas para elementos de concreto não são normalmente modeladas e, portanto, não são apresentadas em tabelas de quantidades geradas por softwares de modelagem. Esses pontos devem ser cuidadosamente analisados quando da adoção de metodologias integradas para a avaliação do desempenho ambiental de um produto da indústria da construção (BUENO; FABRICIO, 2018).

\section{Método}

A avaliação da bibliografia citada como fundamentação demonstrou que existe uma tendência pela utilização de softwares de modelagem como ferramentas de quantificação. Porém, assim como comentam Sacks et al. (2018), existem softwares BIM específicos para tal tarefa, denominadas softwares multifunção e que permitem, além da quantificação, integração e compatibilização de projetos provenientes de diferentes softwares de modelagem, disciplinas e equipes. Tendo isto posto, a seleção de software BIM multifunção adequado foi a primeira etapa dessa pesquisa.

Essa seleção foi realizada tendo como base a adesão de mercado, facilidade de obtenção de licença acadêmica e facilidade de customização e inserção de dados externos. Dadas essas considerações, o software BIM multifunção selecionado foi o Navisworks, desenvolvido e distribuído pela Autodesk. Outro ponto relevante foi a existência da ferramenta nativa Quantification, voltada para o levantamento de quantitativos baseados em composições de serviços similares àquelas encontradas em bases de composições como o SINAPI (CAIXA ECONÔMICA FEDERAL, 2019), sendo, portanto, essa a utilizada como fonte para a quantificação dos materiais utilizado.

Dessa forma, a delimitação da metodologia proposta engloba o tratamento de dados provenientes de bases de composições e inventários de ciclo de vida (LCl), tornando possível sua posterior integração. Uma vez integrado e organizado, passa a ser possível a conversão para formato compatível com o software BIM multifunção escolhido. A Figura 2 apresenta, de forma esquemática, o fluxo das informações. É importante destacar que, apesar da utilização de certos padrões, conforme discutido nos parágrafos seguintes, os métodos utilizados podem ser generalizados para diferentes origens de dados.

A inserção dos dados para o Quantification ocorre a partir de um arquivo no formato eXtensible Markup Language (XML), que deve possuir formato específico contendo as informações necessárias para a correta montagem do catálogo de composições no ambiente do Navisworks. Como exemplo, a Figura 3 demonstra a representação em formato XML para um item de "Alvenaria em bloco vazado de concreto $14 \times 19 \times 39$ " do banco SINAPI. Foge do escopo desse artigo detalhar o funcionamento e estrutura do arquivo XML em questão, portanto, para o completo entendimento da utilização do software e da ferramenta Quantification, os autores recomendam a leitura dos manuais técnicos disponíveis (AUTODESK, 2019). Os autores também entendem que a proposta funciona de forma genérica, passível de adaptação para outros softwares BIM disponíveis no mercado cuja função se assemelha àquela apresentada pelo Navisworks 
SILVA, B. T. R. V.; LIMA, M. G. de

Proposta de integração entre BIM e ACV utilizando composições de serviço

(alguns dos exemplos incluem o Vico Office e o Solibri, conforme apontado por Sacks et al. (2018)), bastando, para isso, que o padrão de inserção de dados seja conhecido.

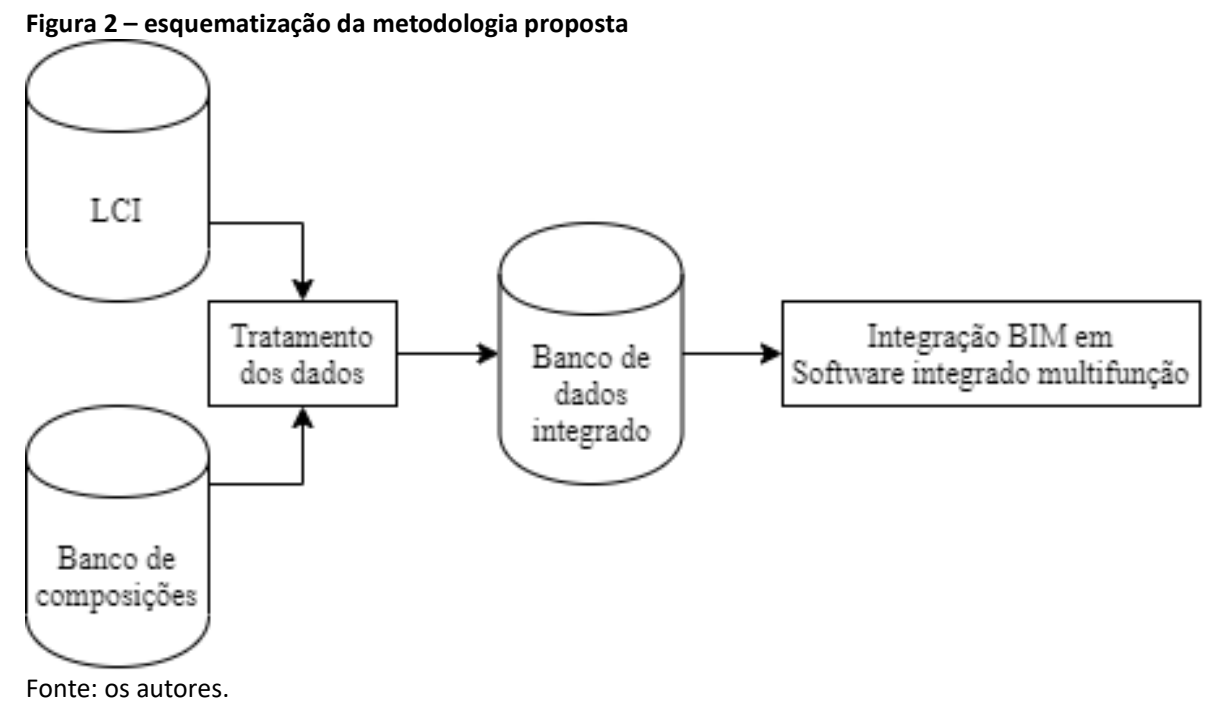

Figura 3 - Entrada de um elemento do tipo item no catálogo do Navisworks

1 <Item Name="ALVENARIA DE VEDAÇ̃̃O DE BLOCOS VAZADOS DE CONCRETO DE 14X19X39CM (ESPESSURA 14CM) [...]" Description="ALVENARIA DE VEDAÇÃO DE BLOCOS VAZADOS DE CONCRETO DE 14X19X39CM (ESPESSURA 14CM) [ . . ] " WBS="87449" Transparency="0.3" Color="2597843" LineThickness="0.1" CatalogId="c48adb68-4a4d-35a4-a0f3-597ef87dfdd2" <Variablecollection>

<Variable Name="Length" Formula="=ModelLength" Units="Meter" />

<Variable Name="Width" Formula="=ModelWidth" Units="Meter" />

<Variable Name="Thickness" Formula="=ModelThickness" Units="Meter" />

<Variable Name="Height" Formula="=ModelHeight" Units="Meter" />

$\langle$ Variable Name="Perimeter" Formula="=ModelPerimeter" Units="Meter" />

<Variable Name="Area" Formula="=ModelArea" Units="SquareMeter" / >

<Variable Name="Volume" Formula="=ModelVolume" Units="CubicMeter" />

<Variable Name="Weight" Formula="=ModelWeight" Units="Kilogram" / >

<Variable Name="Time" Formula="=ModelTime" Units="Hour" />

$\langle$ Variable Name="Distance" Formula="=ModelDistance" Units="Kilometer" />

$<$ Variable Name="Count" Formula="=1" />

$<$ Variable Name="PrimaryQuantity" Formula="=ModelArea" Units="SquareMeter" /> $<$ VariableCollection>

$</$ Item $>$

Fonte: os autores.

\section{Estrutura do banco de dados}

Apesar de ser possível editar o arquivo XML de forma manual, a proposta dessa pesquisa envolve a automatização desse processo. Para isso, foram desenvolvidas rotinas utilizando a linguagem de programação Python (PYTHON SOFTWARE FOUNDATION, 2020) com o auxílio do módulo SQLAlchemy (BAYER, 2012). A utilização dessas rotinas teve como premissa, em um primeiro momento, a criação de um banco de dados contendo as informações necessárias para que, em um segundo momento, as variáveis utilizadas na avaliação de ciclo de vida fossem devidamente convertidas para o formato correto do arquivo XML.

O banco de dados foi criado utilizando Structured Query Language (SQL), que permite uma estruturação mais organizada das informações e facilita o acesso e conversão dos dados. A Figura 4 apresenta o esquema do banco de dados proposto. As tabelas inseridas no banco foram criadas com o objetivo de representar os elementos utilizados para a quantificação do projeto (composições de serviços e recursos) e a relação que 
existe entre estes. Além disso, uma tabela representando a relação entre os recursos e indicadores de impacto de ciclo de vida de fluxos de produtos ou serviços de inventário de ciclo de vida foi também criada. Esses fluxos foram obtidos a partir da consulta do inventário de ciclo de vida disponibilizado pelo ecoinvent (ECOINVENT, 2019). Apesar de a metodologia permitir a inserção de qualquer indicador de impacto (ou outras variáveis quaisquer), para o escopo dessa pesquisa foi utilizado o indicador de midpoint do método de avaliação ReCiPe 2016 para a perspectiva Hierarchist (ReCiPe Midpoint $(H)$ ) para o potencial de aquecimento global em $\mathrm{kg} \mathrm{CO}_{2}$-eq (NATIONAL INSTITUTE FOR PUBLIC HEALTH AND THE ENVIRONMENT, 2018). A escolha pelo método ReCiPe se deu por ser um método global, relativamente recente e recorrente na literatura avaliada. Porém, como o objetivo do estudo não compreende a avaliação de desempenho ambiental de determinados produtos, mesmo que essa tenha sido feita com o objetivo de demonstrar a abordagem proposta, a adoção de outro método de avaliação de impacto pode ser realizada sem que adaptações relevantes precisem ser realizadas.

Figura 4 - esquema do banco de dados sugerido

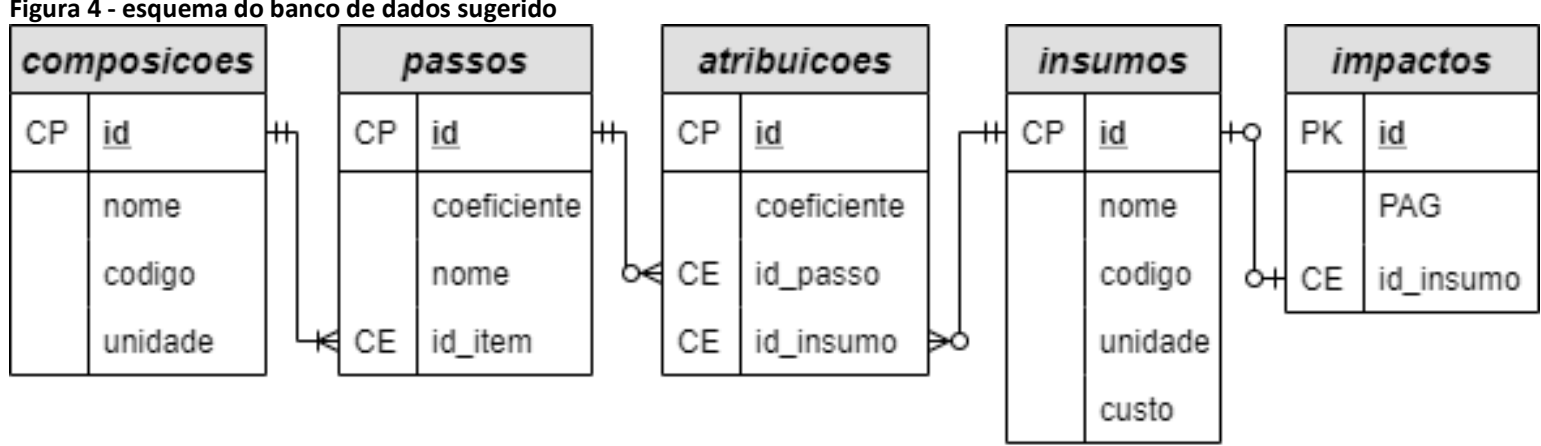

Notas: CP para chave primária (identificador primário); CE para chave externa (identificador relacionado com outra tabela); PAG para Potencial de Aquecimento Global (em kg CO2-eq). Fonte: os autores.

Durante a etapa de construção do banco de dados, a elaboração de rotinas de programação utilizando a linguagem Python objetivou a automatização da leitura dos arquivos contendo as composições de serviço e insumos, disponibilizados pela CAIXA, e dos resultados da aplicação do método ReCiPe Midpoint $H$ (utilizando o software gratuito OpenLCA (GREENDELTA, 2020)) ao inventário de ciclo de vida, ambos convertidos para formato de valores separados por vírgula (CSV), uma vez que foram obtidos em formato de planilhas eletrônicas. As informações obtidas a partir da leitura desses arquivos foram posteriormente carregados para o banco proposto, conforme esquema apresentado na Figura 4. Ou seja, as rotinas responsáveis pela extração dos dados dos arquivos CSV foram desenvolvidas para que a leitura, linha por linha, dos arquivos fosse realizada e as informações pertinentes classificadas e organizadas.

Carregamento dos dados em software multifunção

A elaboração de um banco de dados integrado utilizando a linguagem SQL teve como objetivo a melhor estruturação das informações e, consequentemente, facilitar o acesso, transformação e formatação dos dados, além de permitir que a relação entre as diferentes tabelas (composições, passos, recursos e impactos, conforme Figura 4) fosse melhor estruturada. Porém, conforme evidenciado pela Figura 2, existe a necessidade da mudança de formato para que a interpretação dessas informações pudesse ser realizada pelo software BIM multifunção selecionado, ou seja, como mencionado anteriormente, a ferramenta Quantification do software Navisworks necessita da importação de um arquivo em formato XML cuja estrutura se assemelha àquela apresentada na Figura 3. Outra vantagem em compilar os dados em um banco utilizando a linguagem SQL é a neutralidade, ou seja, as informações estão armazenadas em um 
SILVA, B. T. R. V.; LIMA, M. G. de

Proposta de integração entre BIM e ACV utilizando composições de serviço

formato genérico e conhecido, tornando a conversão para formato diferente daquele requerido pelo Navisworks uma adaptação baseada nos requerimentos do software BIM de interesse, apesar de não comentada nesse estudo.

Isto posto, a conversão das informações para o formato XML adequado à leitura pelo Navisworks também foi automatizado utilizando rotinas de programação. Nesta etapa, a função das rotinas se resume em interpretar as informações compiladas no banco de dados proposto e convertê-las para o formato adequado. A Figura 5 apresenta a relação entre os atributos do banco de dados proposto (à esquerda) e as propriedades do arquivo XML formatado conforme os requisitos para o Quantification para o caso do insumo "Bloco cerâmico de vedação com furos na vertical, 14 X 19 × 39cm" (à direita). A conversão ocorre de forma análoga para as demais tabelas do banco, sendo que, para o completo entendimento dos padrões do arquivo XML, os autores referenciam a documentação do software Navisworks (AUTODESK, 2019).

Figura 5 - Relação entre atributos das tabelas do banco proposto (à esquerda) e elementos e atributos do arquivo XML para o Quantification (à direita). Na figura é apresentada para o caso dos insumos, para demais elementos, a relação ocorreu de forma análoga. PAG indica o potencial de aquecimento global, cuja correspondência foi nomeada como "GlobalWarming" para atender aos padrões de nomenclatura já estabelecidas pelo arquivo

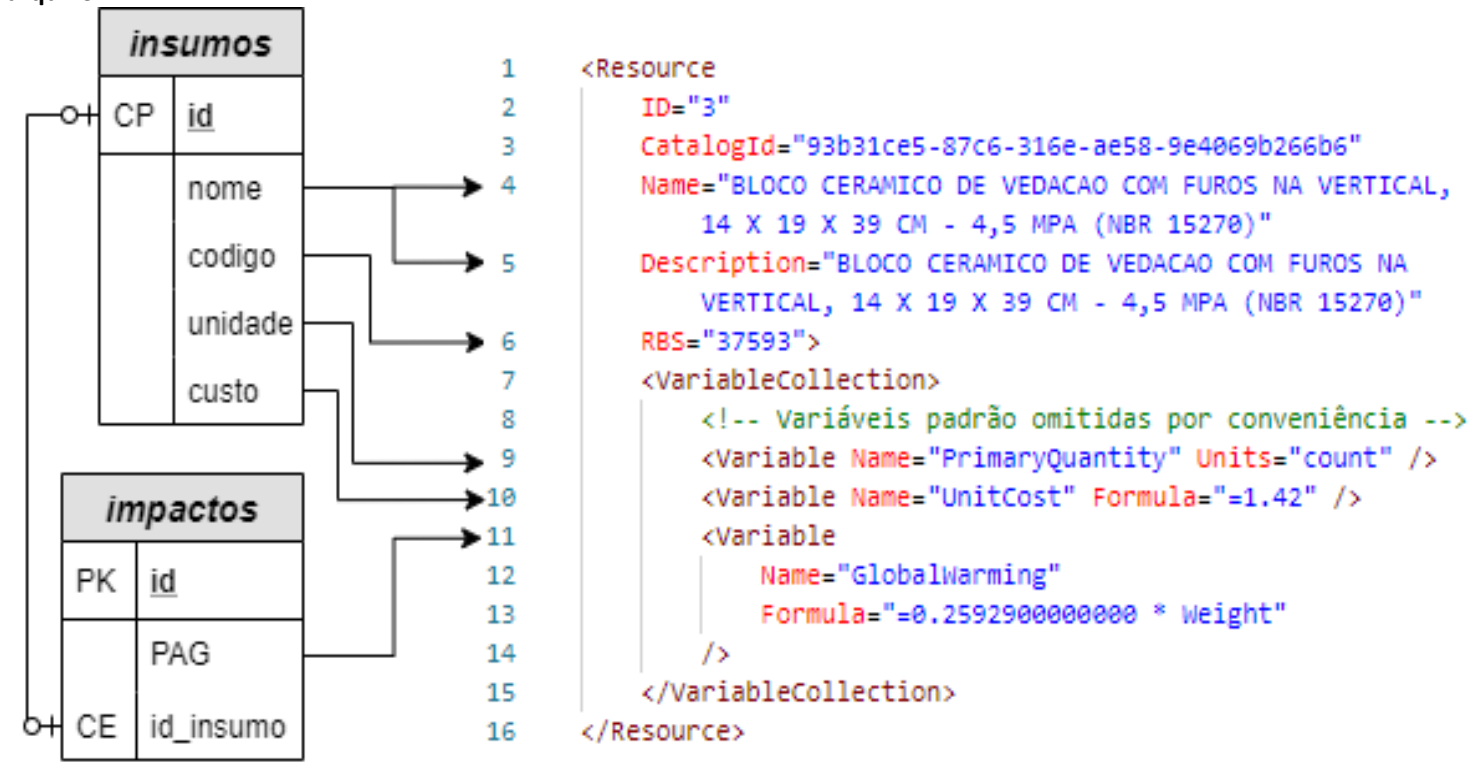

Fonte: os autores.

Algumas considerações sobre o recorte do arquivo XML apresentado à direita na Figura 5:

- Na linha 1, a tag “Resource" indica que o conteúdo representa uma entrada do tipo insumo;

- Na linha 2, o atributo “ID” indica um identificador único entre as outras entradas do tipo "Resource" presentes no arquivo XML;

- Na linha 3, o atributo "CatalogID" representa um identificador único entre todas as entradas do arquivo XML, independentemente do tipo;

- As linhas 2 e 3 representam metadados sobre o recurso em questão e não são apresentados na interface de usuário do Navisworks;

- As linhas 4 e 5 recebem o nome do insumo. Apesar de o atributo da linha 5 indicar uma descrição, nem o banco de dados proposto nem o catálogo do SINAPI apresentam informação correspondente, portanto o nome foi repetido por conveniência; 
- A linha 6 apresenta o código de Estrutura Analítica de Recursos (RBS), recebendo o valor código do banco de dados, sendo que, para o caso apresentado, da utilização do banco SINAPI, o valor do código da composição foi utilizado;

- A tag "VariableCollection" na linha 7 indica o início da coleção de variáveis que serão quantificadas pelo Quantification dentro do Navisworks;

- As linhas 9 a 14 apresentam as variáveis que serão quantificadas, cabendo notar que, assim como comentado na linha 8 da figura, as variáveis padrão disponibilizadas pelo Navisworks foram omitidas.

\section{Aplicação na seleção de materiais}

Para a validação do método apresentado, foi realizado um estudo de caso utilizando dois sistemas de alvenaria cujo núcleo foi modelado tendo como material duas alternativas diferentes. O escopo da avaliação não incluiu as fases de utilização e disposição final ao fim da vida útil esperada dos elementos. A unidade funcional foi o metro quadrado de alvenaria. As alternativas foram criadas conforme indicado pela Figura 6 utilizando software de modelagem BIM e, posteriormente importadas para o ambiente de trabalho do Navisworks.

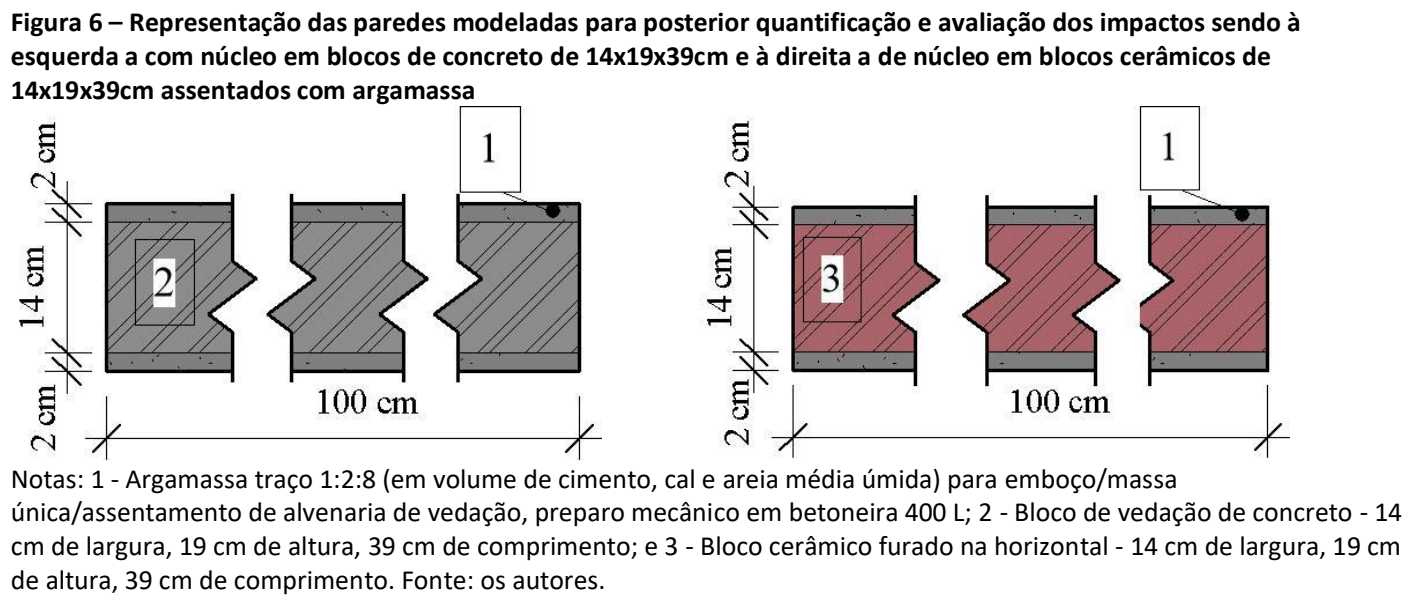

Para a quantificação dos materiais utilizados, as composições de serviços selecionadas foram as obtidas a partir do banco SINAPI de abril de 2020 para o estado de Minas Gerais, e estão indicadas no Quadro 1, assim como os respectivos elementos e funções consideradas. A partir da utilização dessas composições, foi possível prever quais seriam os materiais necessários para a execução dos elementos de alvenaria apresentados na Figura 6. A quantidade de cada recurso foi obtida a partir do levantamento utilizando a ferramenta Quantification.

A Tabela 1 apresenta os processos fornecedores, obtidos a partir da consulta do inventário do ecoinvent, dos recursos considerados para a avaliação de impacto, assim como o respectivo potencial para o indicador de aquecimento global por unidade de medição. Vale notar que a unidade considerada pelo inventário do ecoinvent para um determinado recurso era, por vezes, diferente daquele apresentado pelo SINAPI, portanto, conversões foram realizadas com base na massa específica ou massa por unidade dos materiais, conforme necessidade.

Outra observação que deve ser feita é quanto a falta de representatividade de processos brasileiros em inventários de ciclo de vida. Processos fornecedores da Tabela 
SILVA, B. T. R. V.; LIMA, M. G. de

Proposta de integração entre BIM e ACV utilizando composições de serviço

1 indicados com "BR" ao final representam a forma com que os produtos são obtidos em mercado nacional. Na falta dessa opção, foram selecionadas alternativas de representatividade global, indicadas por "GLO" ou para países emergentes, "RoW".

Quadro 1 - Relação das composições utilizadas para a quantificação dos materiais

\begin{tabular}{|c|c|c|}
\hline Parede & Composição SINAPI & Função \\
\hline $\begin{array}{l}\text { Com núcleo em } \\
\text { bloco cerâmico }\end{array}$ & $\begin{array}{l}\text { ALVENARIA DE VEDAÇÃO DE BLOCOS VAZADOS DE CONCRETO DE 14X19X39CM (ESPESSURA 14CM) } \\
\text { DE PAREDES COM ÁREA LÍQUIDA MENOR QUE 6M² SEM VÃOS E ARGAMASSA DE ASSENTAMENTO } \\
\text { COM PREPARO EM BETONEIRA. AF_06/2014 }\end{array}$ & Núcleo \\
\hline $\begin{array}{l}\text { Com núcleo em } \\
\text { bloco de concreto }\end{array}$ & $\begin{array}{l}\text { ALVENARIA DE VEDAÇÃO DE BLOCOS CERÂMICOS FURADOS NA VERTICAL DE 14X19X39CM } \\
\text { (ESPESSURA 14CM) DE PAREDES COM ÁREA LÍQUIDA MENOR QUE 6M² SEM VÃOS E ARGAMASSA DE } \\
\text { ASSENTAMENTO COM PREPARO EM BETONEIRA. AF_06/2014 }\end{array}$ & Núcleo \\
\hline \multirow[t]{2}{*}{ Comum } & $\begin{array}{c}\text { ARGAMASSA TRAÇO 1:2:8 (EM VOLUME DE CIMENTO, CAL E AREIA MÉDIA ÚMIDA) PARA } \\
\text { EMBOÇO/MASSA ÚNICA/ASSENTAMENTO DE ALVENARIA DE VEDAÇÃO, PREPARO MECÂNICO COM } \\
\text { BETONEIRA } 400 \text { L. AF_08/2019 }\end{array}$ & $\begin{array}{c}\text { Assentamento/ } \\
\text { Revestimento }\end{array}$ \\
\hline & $\begin{array}{l}\text { TRANSPORTE COM CAMINHÃO BASCULANTE DE } 10 \text { M3, EM VIA URBANA PAVIMENTADA, DMT } \\
\text { ACIMA DE } 30 \mathrm{KM} \text { (UNIDADE: TXKM). AF 04/2016 }\end{array}$ & $\begin{array}{l}\text { Transporte dos } \\
\text { insumos }\end{array}$ \\
\hline
\end{tabular}

Fonte: os autores.

Cabe ressaltar que, uma vez carregados no Navisworks, os valores podem ser facilmente visualizados e editados a partir da interface de usuário disponibilizada pelo software. A Figura 7 demonstra como os recursos, uma vez carregados, se apresentam em forma de lista e como a variável criada para representar o valor do indicador de aquecimento global (GlobalWarming) se apresenta nos detalhes de um recurso selecionado, como, por exemplo, o bloco cerâmico.

O levantamento das quantidades de cada recurso ocorreu seguindo conforme indica a Autodesk (2019). Para isso, basta selecionar o elemento cujas quantidades se deseja calcular e associá-lo ao item de catálogo, ou composição, correspondente. É possível, ainda, associar um elemento a mais de um item, ou seja, o mesmo elemento modelado pode ser quantificado para os serviços de assentamento de blocos e transporte, por exemplo, como demonstrado pela Figura 8.

Figura 7 - Apresentação do recurso para o bloco cerâmico na lista de recursos carregados no Quantification a esquerda e, a direita, os detalhes do recurso, incluindo a variável GlobalWarming criada para representar o indicador de potencial de aquecimento global

\section{Resources}

- ßAREIA MEDIA - POSTO JAZIDA/FORNECEDOR (RETIRADO NA JAZID...

- 3 BLOCO CERAMICO DE VEDACAO COM FUROS NA VERTICAL, 14 X 1.

- ßBLOCO VEDACAO CONCRETO 14 X 19 X 39 CM (CLASSE C - NBR 61.

- CAL HIDRATADA CH-I PARA ARGAMASSAS

- 3 CAMINHAO TRUCADO, PESO BRUTO TOTAL 23000 KG, CARGA UTIL...

- CIMENTO PORTLAND COMPOSTO CP ॥-32

- 3 ENERGIA ELETRICA ATE 2000 KWH INDUSTRIAL, SEM DEMANDA

- BOLEO DIESEL COMBUSTIVEL COMUM Resource Name Resource Breakdown Structure

BLOCO CERAMICO DE VEDACAO COM FUROS NA 37593

Description

BLOCO CERAMICO DE VEDACAO COM FUROS NA VERTICAL, $14 \times 19 \times 39$ CM - 4,5 MPA (NBR 15270)

Resource Calculations

\begin{tabular}{|crc|}
\hline Variable & Formula & Units \\
GlobalWarming & $=0.25929^{*}$ Weight & Kilogram
\end{tabular}

Fonte: os autores. 
SILVA, B. T. R. V.; LIMA, M. G. de

Proposta de integração entre BIM e ACV utilizando composições de serviço

Tabela 1 - Relação dos recursos considerados para a avaliação de impacto e o valor do indicador de potencial de aquecimento global conforme ReCiPe Midpoint $(H)$

\begin{tabular}{|c|c|c|c|c|}
\hline \multirow{2}{*}{ Recurso (SINAPI) } & \multirow{2}{*}{ Processo fornecedor (ecoinvent) } & \multirow{2}{*}{$\begin{array}{l}\text { Potencial para } \\
\text { aquecimento global } \\
\text { (kg CO} 2 \text {-eq) }\end{array}$} & \multicolumn{2}{|c|}{ Unidade } \\
\hline & & & SINAPI & ecoinvent \\
\hline $\begin{array}{l}\text { Areia media - posto jazida/fornecedor (retirado } \\
\text { na jazida, sem transporte) }\end{array}$ & $\begin{array}{c}\text { silica sand production | silica sand | } \\
\text { APOS, S-RoW }\end{array}$ & 0,04274 & $\mathrm{~m}^{3}$ & $\mathrm{Kg}$ \\
\hline $\begin{array}{l}\text { Bloco vedação concreto } 14 \times 19 \times 39 \mathrm{~cm} \text { (classe } \\
\text { C - NBR 6136) }\end{array}$ & $\begin{array}{c}\text { concrete block production | concrete } \\
\text { block |APOS, U - BR }\end{array}$ & 0,06854 & un & $\mathrm{Kg}$ \\
\hline Cal hidratada $\mathrm{CH}-\mathrm{I}$ para argamassas & $\begin{array}{c}\text { lime production, hydrated, packed I } \\
\text { lime, hydrated, packed | APOS, U - } \\
\text { RoW }\end{array}$ & 0,94625 & $\mathrm{Kg}$ & $\mathrm{Kg}$ \\
\hline Cimento Portland composto CP II-32 & $\begin{array}{l}\text { cement production, Portland | } \\
\text { cement, Portland| APOS, U - BR }\end{array}$ & 0,83739 & $\mathrm{Kg}$ & $\mathrm{Kg}$ \\
\hline $\begin{array}{l}\text { Energia elétrica até } 2000 \text { kWh industrial, sem } \\
\text { demanda }\end{array}$ & $\begin{array}{l}\text { market for electricity, low voltage | } \\
\text { electricity, low voltage | APOS, U - BR, } \\
\text { South-eastern grid }\end{array}$ & 0,26326 & $\mathrm{kWh}$ & kWh \\
\hline Óleo diesel combustível comum & $\begin{array}{c}\text { market for diesel | diesel | APOS, U - } \\
\text { BR }\end{array}$ & 0,48371 & $\mathrm{Kg}$ & $\mathrm{Kg}$ \\
\hline $\begin{array}{l}\text { Bloco cerâmico de vedação com furos na } \\
\text { vertical, } 14 \text { × } 19 \times 39 \mathrm{~cm}-4,5 \mathrm{mPa} \text { (NBR 15270) }\end{array}$ & $\begin{array}{c}\text { clay brick production | clay brick | } \\
\text { APOS, U -RoW }\end{array}$ & 0,25929 & un & $\mathrm{Kg}$ \\
\hline $\begin{array}{l}\text { Caminhão trucado, peso bruto total } 23000 \mathrm{~kg} \text {, } \\
\text { carga útil máxima } 15935 \mathrm{~kg} \text {, distância entre } \\
\text { eixos } 4,80 \text { m, potência } 230 \mathrm{cv} \text { (inclui cabine e } \\
\text { chassi, não inclui carroceria) }\end{array}$ & $\begin{array}{l}\text { market for lorry, } 16 \text { metric ton | lorry, } \\
16 \text { metric ton | APOS, U - GLO }\end{array}$ & 23860,4 & un & item \\
\hline
\end{tabular}

Fonte: os autores.

Figura 8 - Seleção do núcleo em bloco cerâmico (destacado em azul) demonstrando seu material, na janela Properties, e os itens de catálogo cujas quantidades são obtidas a partir das propriedades do elemento, na janela Select Workbook Item

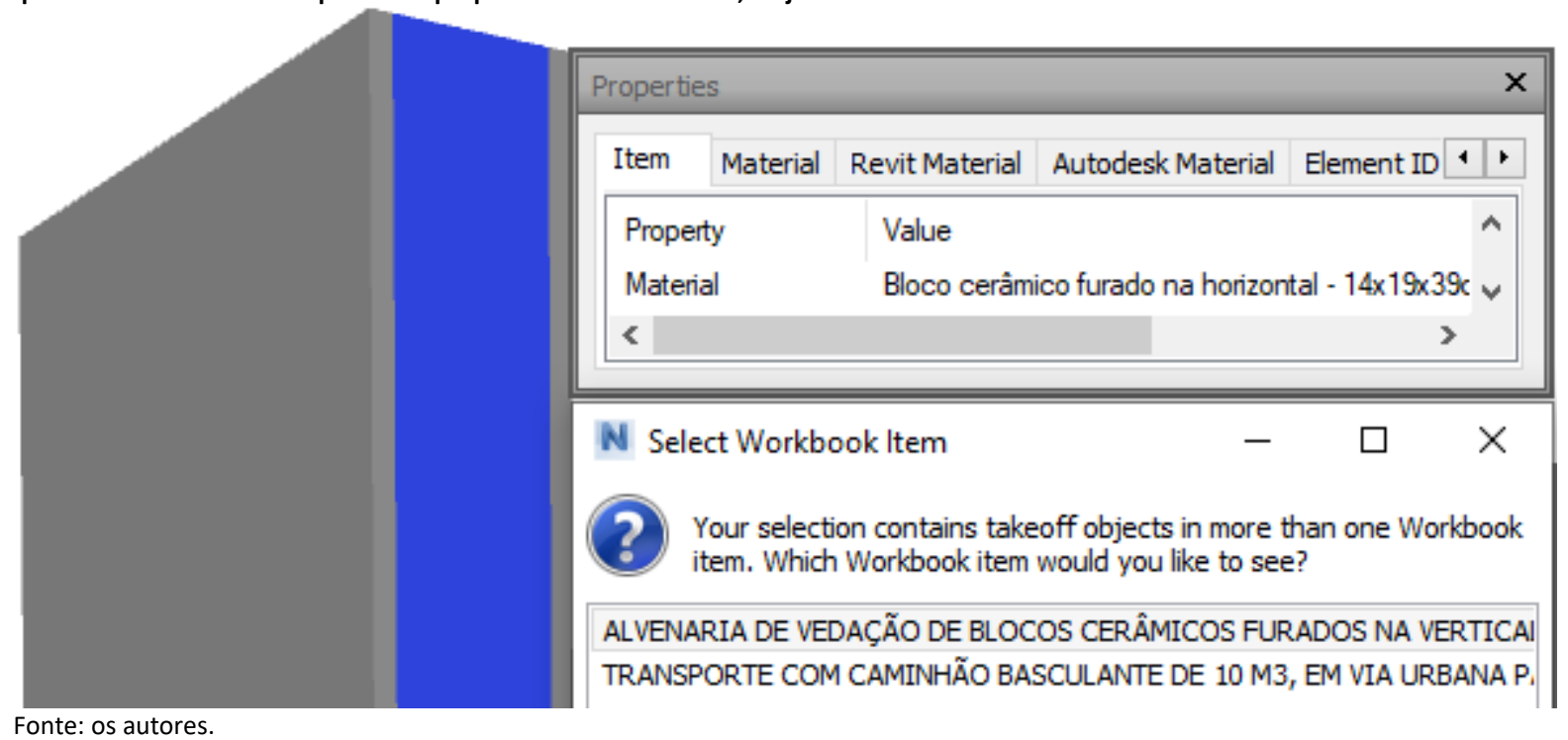

Fonte: os autores.

\section{Resultados e discussão}

A partir da aplicação da metodologia proposta para a realização da avaliação de impacto de ciclo de vida para os elementos, conforme discutido no estudo de caso, foi possível gerar relatório automatizados a partir da quantificação obtida diretamente de modelos BIM. A abordagem proposta se apresentou como uma forma flexível de realizar o levantamento dos impactos associados aos diferentes materiais utilizados além de não ocultar do usuário quais são os valores que estão sendo considerados. A utilização do Building Information Modeling tem apresentado resultados positivos quando empregado para auxiliar o levantamento de custos de construções (VIGNEAULT et al., 2019). Não diferente, a sua utilização para o cálculo de indicadores de desempenho ambiental se apresentou como uma abordagem viável, apesar das adaptações necessárias, o que pode ser justificado pelo caráter genérico que as ferramentas 
disponibilizadas por softwares BIM apresentam, ou seja, os desenvolvedores normalmente oferecem plataformas para que os usuários criem personalização adaptadas à determinados fluxos de trabalho.

A comparação entre os valores apresentados pelo indicador de impacto relacionado com o potencial de aquecimento global das duas alternativas de alvenaria estudadas permitiu demonstrar que a utilização de blocos cerâmicos resulta em um desempenho relativamente reduzido da alvenaria, quando comparado com blocos em concreto, conforme apresentado na Figura 9 a esquerda. Cabe observar que, mesmo a massa dos blocos de concreto seja relativamente superior àquela dos blocos cerâmicos, conforme demonstrado pela Figura 9 a direita, tornando necessária uma maior energia no transporte, a maior diferença ainda está associada à produção e utilização dos diferentes tipos de blocos. Conforme apontado por Bueno et al. (2016), isso pode estar associado à queima dos elementos cerâmicos necessária para a cura, emitindo, assim, uma grande quantidade de gases de efeito estufa. Destaca-se que, conforme comentado anteriormente, existe pouca representatividade dos processos brasileiros em inventários de ciclo de vida, fazendo com que fosse necessária a adoção de dados secundários. Apesar do objetivo desse estudo não ser a realização de uma avaliação de desempenho de materiais, e sim a apresentação de uma metodologia para tal, é importante ressaltar que os resultados obtidos a partir do estudo de caso apresentado pode não condizer com a realidade, uma vez que adaptações e considerações por vezes necessárias não foram realizadas (SILVA et al., 2020).

Figura 9 - apresentação dos resultados obtidos a partir do estudo de caso para o Potencial de Aquecimento Global (a
esquerda) e comparação entre a massa total das camadas construtivas da parede (a direita)
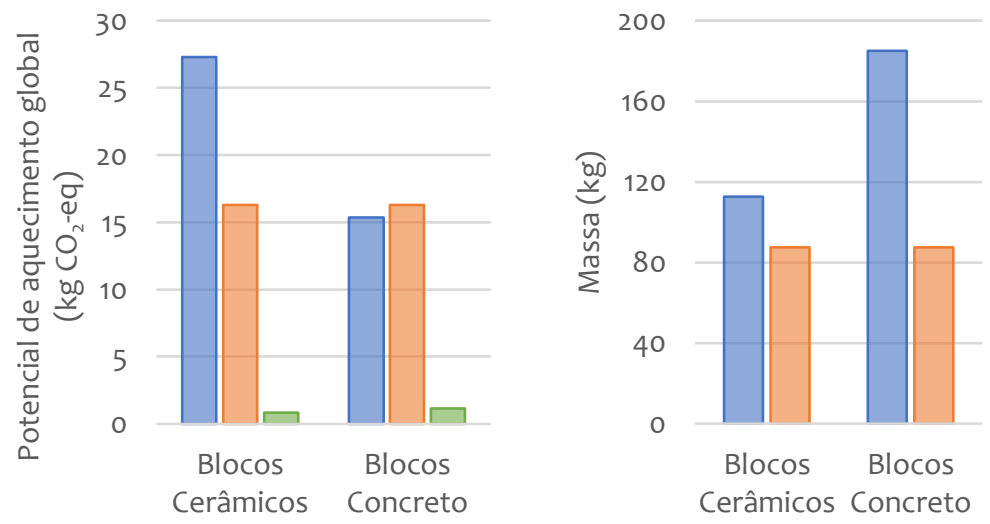

$\square$ Núcleo $\square$ Argamassa $\square$ Transporte

Fonte: os autores.

De forma complementar, uma vez que as composições de serviço SINAPI utilizadas para o levantamento de materiais têm como premissa a utilização como ferramentas de auxílio para a estimativa de custos da construção, foi possível avaliar indicadores de custos das alternativas apresentadas no estudo de caso de forma paralela ao indicador de potencial de aquecimento global. Conforme demonstrado pela Figura 10, o custo de alvenarias em bloco de concreto é relativamente superior ao previsto para alvenarias que utilizam blocos cerâmicos. Portanto, quando tomadores de decisão levam em consideração apenas o custo de materiais, soluções menos sustentáveis podem ser selecionadas. 
SILVA, B. T. R. V.; LIMA, M. G. de

Proposta de integração entre BIM e ACV utilizando composições de serviço

Figura 10 - Estimativa de custos para os diferentes serviços previstos na execução das alvenarias propostas no estudo de caso

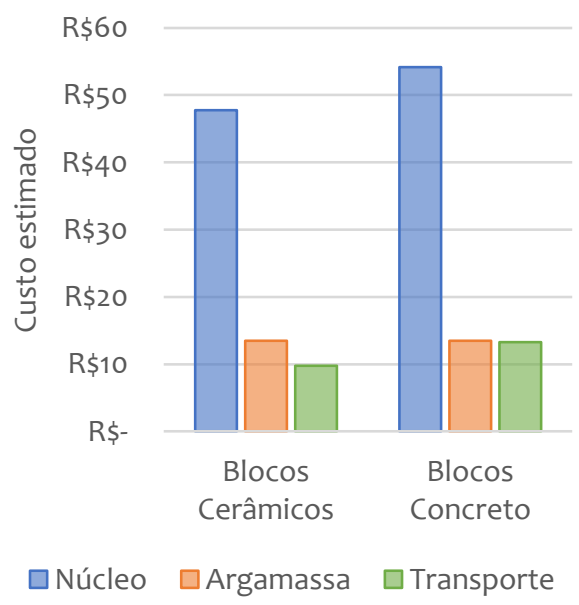

Fonte: os autores.

A complexidade da realização de ACV para elementos construtivos era fator limitante para a utilização de dados sobre impactos ambientais por arquitetos, engenheiros e construtoras (SOUST-VERDAGUER; LLATAS; GARCÍA-MARTÍNEZ, 2016). A utilização de softwares BIM permitiu que os dados estivessem facilmente organizados e ao alcance do avaliador, bastando realizar a quantificação de forma equivalente a um levantamento de custos, cuja realização já ocorre de forma habitual para obras de construção civil. Porém, vale ressaltar que ainda existe uma necessidade de consolidação e validação de inventários de ciclo de vida de forma que a realidade das obras brasileiras possa ser devidamente representada.

Por fim, o estudo utilizou as rotinas desenvolvidas para aplicar a metodologia para o caso apresentado no capítulo "Aplicação na seleção de materiais", fazendo com que o banco de dados em formato SQL apresentasse um tamanho de arquivo total de 64kb, representando as composições apresentadas no Quadro 1. Para a compilação de toda a base SINAPI, que possui cerca de 6400 composições e 5300 insumos, o banco de dados apresentou $7.964 \mathrm{~kb}$ de tamanho. A conversão para o arquivo XML, para o caso das composições apresentadas no Quadro 1 gerou um arquivo de aproximadamente $240 \mathrm{~kb}$ e, 51Mb para o banco SINAPI completo. Vale destacar que, uma vez que está sendo apresentada a primeira interação para realizar esse tipo de operação pelos autores, ainda pode existir espaço para otimização, como uma melhor estruturação do banco de dados proposto ou o desenvolvimento de interfaces de usuário que facilitariam a interação com as rotinas desenvolvidas.

\section{Conclusão}

O desenvolvimento da metodologia proposta permitiu a integração entre diferentes fontes de informação que tem como objetivo a quantificação de indicadores de desempenho de um produto da indústria da construção. Seja a partir do levantamento de custos ou pela avaliação de indicadores ambientais, o método proposto possibilitou que um modelo tridimensional fosse utilizado para a automatização na extração das quantidades. Porém, ainda se faz necessária a utilização do que foi apresentada nesse estudo em instalações mais complexas, apesar de os autores visualizarem que as limitações se encontrariam na qualidade e disponibilidade de dados confiáveis em inventários de ciclo de vida ou bancos de composições de serviço. 
Apesar de não apresentado nesse estudo, é possível utilizar qualquer indicador de avaliação de ciclo de vida para alimentar o banco de dados e, posteriormente, o catálogo do software BIM utilizado. Portando, seria interessante avaliar, em estudos futuros, quais seriam as variáveis mais adequadas para serem utilizadas de forma que o modelo BIM possa ser devidamente utilizado para tomadas de decisão no sentido de melhorar o desempenho ambiental de produtos da indústria da construção.

Softwares BIM possuem finalidades bem definidas. Portanto, é processo relevante identificar quais são os softwares melhor adaptados para uma determinada tarefa, ou seja, os autores recomendam que, durante o desenvolvimento de estudos envolvendo tecnologia BIM, a etapa de identificação de software adequado seja sempre levada em consideração. Dessa forma, espera-se que exista uma redução na quantidade de adaptações necessárias, tornando menos inviável a integração com fluxos de trabalhos distinto.

A utilização do software Navisworks demonstrou que existe potencial para quantificação dos elementos presentes no projeto desde suas fases iniciais, uma vez que é possível atribuir composições e recursos a elementos com baixos níveis de desenvolvimentos, tornando possível que, mesmo de forma preliminar, elementos provisórios, utilizados apenas para definições iniciais de formas, volumetrias etc. possam ser considerados em avaliações precoces de desempenho. Porém, assim como demonstrado por Rezaei, Bulle e Lesage (2019), a utilização de ferramentas para a estimativa de quantitativos de projeto tende a apresentar resultados mais precisos conforme o nível de detalhamento aumenta, o que não descarta a necessidade de sua utilização desde fases iniciais. Para estudos futuros, a manifestações de incertezas causadas por um baixo nível de desenvolvimento de projetos ainda nas fases iniciais de elaboração pode ser levada em consideração, demonstrando como a definição de materiais e formas mais bem definidas poderiam contribuir para a identificação de possíveis otimizações no projeto e aumentar o desempenho do produto em avaliação.

\section{Agradecimentos}

O presente trabalho foi realizado com apoio do CNPq, Conselho Nacional de Desenvolvimento Científico e Tecnológico - Brasil. Processo 133678/2019-9

\section{Notas}

(1) O código fonte desenvolvido encontra-se disponibilizado em repositório aberto na plataforma Github, acessível pela url https://github.com/brunotvs/projecLCQ_paper.

\section{Referências}

AUTODESK. Produtos Navisworks | Autodesk Knowledge Network. Disponível em: https://knowledge.autodesk.com/pt-br/support/navisworksproducts? $p=N A V \& v=2021 \&$ sort $=$ score $\&$ page $=1 \&$ knowledgeSource $=$ Documenta $\%$ C $3 \% A 7 \%$ C $3 \% A 30 \% 20$ do\%20produto . Acesso em: 13 maio. 2020.

AZHAR, S. Building information modeling (BIM): trends, benefits, risks, and challenges for the AEC industry. Leadership and Management in Engineering, v. 11, n. 3, p. 241-252, 2011.

BARLISH, K.; SULLIVAN, K. How to measure the benefits of BIM-A case study approach. Automation in Construction, v. 24, p. 149-159, 2012.

BARROS, N. N.; SILVA, V. G. DA. BIM na avaliação do ciclo de vida de edificações: revisão da literatura e estudo comparativo. PARC Pesquisa em Arquitetura e Construção, v. 7, n. 2, p. 89-101, 2016. 
SILVA, B. T. R. V.; LIMA, M. G. de

Proposta de integração entre BIM e ACV utilizando composições de serviço

BAYER, M. SQLAIchemy. In: BROWN, A.; WILSON, G. (Eds.). The architecture of open source applications. volume II: structure, scale, and a few more fearless hacks. Mountain View: aosabook.org, 2012. cap. 20, p 291-314.

BUENO, C. et al. Sensitivity analysis of the use of Life Cycle Impact Assessment methods: a case study on building materials. Journal of Cleaner Production, v. 112, p. 2208-2220, 2016. DOI: https://doi.org/10.1016/j.jclepro.2015.10.006

BUENO, C.; FABRICIO, M. M. Comparative analysis between a complete LCA study and results from a BIM-LCA plugin. Automation in Construction, v. 90, p. 188-200, 2018. DOI: https://doi.org/10.1016/j.autcon.2018.02.028

CAIXA ECONÔMICA FEDERAL. SINAPI: metodologias e conceitos. 6. ed. Brasília, DF: Caixa, 2019.

ECOINVENT. Ecoinvent. Disponível em: https://www.ecoinvent.org/. Acesso em: 16 out. 2019.

GENG, S. et al. Building Life Cycle Assessment research: a review by bibliometric analysis. Renewable and Sustainable Energy Reviews, v. 76, p. 176-184, 2017. DOI: https://doi.org/10.1016/j.rser.2017.03.068

GREENDELTA. openLCA.org | openLCA is a free, professional Life Cycle Assessment (LCA) and footprint software with a broad range of features and many available databases, created by GreenDelta since 2006, 2020. Disponível em: http://www.openlca.org/. Acesso em: 27 maio. 2020

HU, Z.; ZHANG, J.; DENG, Z. Construction process simulation and safety analysis based on building information model and 4D technology. Tsinghua Science and Technology, v. 13, n. S1, p. 266-272, 2008. DOI: https://doi.org/10.1016/S1007-0214(08)70160-3

ILHAN, B.; YAMAN, H. Green Building Assessment Tool (GBAT) for integrated BIM-based design decisions. Automation in Construction, v. 70, p. 26-37, 2016. DOI: https://doi.org/10.1016/j.autcon.2016.05.001

INTERNATIONAL ORGANIZATION FOR STANDARDIZATION. ISO 14044:2006: environmental management, Life Cycle Assessment, requirements and guidelines. Geneva, Suiça: ISO, $2006 a$.

INTERNATIONAL ORGANIZATION FOR STANDARDIZATION. ISO 14040:2006: environmental management — Life Cycle Assessment — Principles and framework. Geneva, Suiça: ISO, $2006 \mathrm{~b}$.

INYIM, P.; RIVERA, J.; ZHU, Y. Integration of Building Information Modeling and economic and environmental impact analysis to support sustainable building design. Journal of Management in Engineering, v. 31, n. 1, p. A4014002, 2015. DOI: https://doi.org/10.1061/(ASCE)ME.1943-5479.0000308

KEHILY, D. Embedding life cycle costing in 5D BIM. Journal of Information Technology in Construction, v. 22, pg. 145-167. 2017.

KENSEK, K. M.; KUMAR, S. Sustainable design through interoperability: BIM and energy analysis programs, a case study. Cadernos de Pós-graduação em Arquitetura e Urbanismo, v. 8, n. 1, p. 42-58, 2008.

NATIONAL INSTITUTE FOR PUBLIC HEALTH AND THE ENVIRONMENT. LCIA: the ReCiPe model | RIVM. Disponível em: https://www.rivm.nl/en/life-cycle-assessment-Ica/recipe. Acesso em: 27 maio. 2020.

NATIONAL INSTITUTE OF BUILDING SCIENCES. About the National BIM Standard-United States ${ }^{\circledR} \mid$ National BIM Standard - United States. Disponível em: https://www.nationalbimstandard.org/about. Acesso em: 8 out. 2019.

NEATH, S.; HULSE, R.; CODD, A. Building Information Modelling in practice: transforming Gatwick airport, UK. Proceedings of the Institution of Civil Engineers, v. 167, n. 2, p. 81--87, 2014. DOI: https://doi.org/10.1680/cien.13.00018

ORTIZ, O.; CASTELLS, F.; SONNEMANN, G. Sustainability in the construction industry: a review of recent developments based on LCA. Construction and Building Materials, v. 23, n. 1, p. 28-39, 2009. DOI:

https://doi.org/10.1016/j.conbuildmat.2007.11.012 
SILVA, B. T. R. V.; LIMA, M. G. de

Proposta de integração entre BIM e ACV utilizando composições de serviço

OTI, A. et al. Structural sustainability appraisal in BIM. Automation in Construction, v. 69, p. 44-58, 2016. DOI: https://doi.org/10.1016/j.autcon.2016.05.019

PYTHON SOFTWARE FOUNDATION. Welcome to python.org. Disponível em: https://www.python.org/. Acesso em: 18 mar. 2020.

RADKE, A. M.; WALLMARK, T.; TSENG, M. M. An automated approach for identification and resolution of spatial clashes in building design. In: INTERNATIONAL CONFERENCE ON INDUSTRIAL ENGINEERING AND ENGINEERING MANAGEMENT. 2009, Hong Kong. Proceedings [...]. Hong Kong: IEEE, dez. 2009. DOI:

https://doi.org/10.1109/IEEM.2009.5373167

REZAEI, F.; BULLE, C.; LESAGE, P. Integrating Building Information Modeling and Life Cycle Assessment in the early and detailed building design stages. Building and Environment, v. 153, p. 158-167, 2019. DOI:

https://doi.org/10.1016/j.buildenv.2019.01.034

SACKS, R. et al. BIM handbook: a guide to Building Information Modeling for owners, designers, engineers, contractors, and facility managers. 3 edition ed. Hoboken, New Jersey: Wiley, 2018.

SILVA, F. B. et al. Avaliação do ciclo de vida do concreto dosado em central com base em dados da indústria brasileira. Concreto \& Construções, v. XLVIII, n. 98, p. 91-97, 2020. DOI: http://dx.doi.org/10.4322/1809-

7197.2020.98.0010

SIMAPRO. SimaPro|The world's leading LCA software. Disponível em: https://simapro.com/. Acesso em: 28 maio. 2020.

SOUST-VERDAGUER, B.; LLATAS, C.; GARCÍA-MARTÍNEZ, A. Simplification in Life Cycle Assessment of single-family houses: a review of recent developments. Building and Environment, v. 103, p. 215-227, 2016. DOI:

https://doi.org/10.1016/j.buildenv.2016.04.014

SOUST-VERDAGUER, B.; LLATAS, C.; GARCÍA-MARTÍNEZ, A. Critical review of BIM-based LCA method to buildings. Energy and Buildings, v. 136, p. 110-120, 2017. DOI: https://doi.org/10.1016/j.enbuild.2016.12.009

SPHERA. Life Cycle Assessment LCA software. Disponível em: http://www.gabi-software.com/brazil/index/. Acesso em: 28 maio. 2020.

THE AMERICAN INSTITUTE OF ARCHTECTS. Integrated project delivery: a guide. 1. ed. California: AIA California Council, 2007.

VIGNEAULT, M.-A. et al. An innovative framework of 5D BIM solutions for construction cost management: a systematic review. Archives of Computational Methods in Engineering, p. 1-18, 2019. DOI:

http://dx.doi.org/10.1007/s11831-019-09341-z

WONG, J. K. W.; ZHOU, J. Enhancing environmental sustainability over building life cycles through green BIM: a review. Automation in Construction, v. 57, p. 156-165, 2015.

\section{Bruno Thiago Rego Valeriano Silva}

Engenheiro Civil. Mestrando em Engenharia de Infraestrutura Aeronáutica. Endereço postal: Rua Constante Marcassa, 88, Poços de Caldas, MG, Poços de Caldas, 37701-187

2 Maryangela Geimba de Lima

Engenheira Civil. Doutora em Engenharia Civil. Professora Associada. Endereço postal: Diretoria Cta, Campus do CTA, São José dos Campos, SP, Brasil, 12228-901 